\title{
ESTUDIOS
}

\section{Acerca del carácter ontológico del esquematismo trascendental}

\author{
On the ontological character \\ of transcendental schematism
}

\author{
MARTÍN ARIAS-ALBISU \\ Consejo Nacional de Investigaciones Científicas y Técnicas (Argentina)
}

Recibido: 20-02-2011

Aprobado definitivamente: 05-05-2011

\begin{abstract}
RESUMEN
El objetivo de este artículo es demostrar que la doctrina kantiana del esquematismo trascendental trata una problemática ontológica. Con ese fin, en primer lugar, estudiamos las relaciones entre categorías, esquemas y principios del entendimiento. De esta manera señalamos la íntima relación entre los esquemas y los principios. En segundo lugar, mostramos que el sistema de los principios del entendimiento puede entenderse como un tratado de ontología. En tercer lugar, presentamos y criticamos un grupo de interpretaciones semánticas del esquematismo trascendental.
\end{abstract}

PALABRAS CLAVE

KANT, FILOSOFÍA TRASCENDENTAL, ESQUEMATISMO, ONTOLOGÍA.

\begin{abstract}
The aim of this paper is to demonstrate that the Kantian doctrine of transcendental schematism deals with an ontological problematic. With this purpose in mind, in the first place, I study the relationships between categories, schemata and principles of understanding. In this way I point out the close relationship between schemata and principles. In the second place, I demonstrate that the system of the principles of understanding can be conceived as an ontological treatise. In the third place, I present and review a group of semantic interpretations of transcendental schematism.
\end{abstract}

\section{KEYWORDS}

KANT, TRANSCENDENTAL PHILOSOPHY, SCHEMATISM, ONTOLOGY.

(C) Contrastes. Revista Internacional de Filosofía, vol. XVII (2012), pp. 7-25. ISSN: 1136-4076

Departamento de Filosofía, Universidad de Málaga, Facultad de Filosofía y Letras Campus de Teatinos, E-29071 Málaga (España) 
EL OBJETIVO DEL PRESENTE ARTículo es mostrar que la doctrina kantiana del esquematismo trascendental trata una problemática de carácter ontológico. Con este fin, en primer lugar, estudiaremos las relaciones entre las categorías, los esquemas trascendentales introducidos en el «Capítulo del esquematismo» ${ }^{1}$ y los principios del entendimiento demostrados en el «Sistema de los principios».2 Mostraremos que los esquemas y los principios están íntimamente relacionados. En segundo lugar, indicaremos que el «Sistema de los principios» puede entenderse como un tratado de ontología. Por último, presentaremos y criticaremos una corriente de interpretación diferente de la nuestra, a saber, aquella según la cual el esquematismo trascendental efectúa una interpretación de las categorías. «Interpretación» tiene aquí aproximadamente el sentido que posee el término en la semántica lógica. De esta manera obtendremos evidencia indirecta de la corrección de nuestra interpretación ontológica.

Señalemos que si bien la interpretación ontológica del esquematismo trascendental no es novedosa, ${ }^{3}$ sí lo es la forma en que la demostramos y la manera en que presentamos las relaciones entre categorías, esquemas trascendentales y principios de entendimiento.

Antes de comenzar, introducimos brevemente la problemática del esquematismo trascendental. El objetivo más general del «Capítulo del esquematismo» es especificar las condiciones bajo las cuales es posible la aplicación de las distintas categorías a la multiplicidad contenida en las intuiciones empíricas. Esta aplicación es necesaria a fin de que la multiplicidad empírica sea constituida como objeto empírico de una experiencia humana única. El estudio de las condiciones mencionadas, es decir, los esquemas trascendentales, se torna necesario en virtud de que la heterogeneidad que se presenta entre las catego-

1 El capítulo de la Crítica de la razón pura (Kritik der reinen Vernunft: $K r V$ ) al que hacemos referencia con la expresión «Capítulo del esquematismo» se denomina «Del esquematismo de los conceptos puros del entendimiento» ( $c f$. A 137/B 176 ss.). Remitimos a la Crítica de la razón pura según la paginación de la edición original ( $\mathrm{A}$ » hace referencia a la primera edición de 1781, y «B» a la segunda de 1787). Citamos esta obra de acuerdo con la traducción de M. Caimi: Crítica de la razón pura. Buenos Aires: Colihue, 2007. En cuanto a las fuentes, nos hemos servido de los textos incluidos en Kant, I.: Kant's gesammelte Schriften. Berlín: hrsg. von der Königlich Preußischen Akademie der Wissenschaften, 1902 ss. Remitimos a esta edición de conjunto con la sigla «AA» (Akademie-Ausgabe) y damos a continuación el número de tomo (en romanos) y de página (en arábigos). Cuando hacemos referencia a la sección de una obra sin mencionar esta última, ella pertenece a la Crítica de la razón pura (por ejemplo: «Analogías de la experiencia»).

2 El capítulo de $K r V$ al que nos referimos con la expresión «Sistema de los principios» se denomina «Sistema de todos los principios del entendimiento puro» ( $c f$. A 148/B 187 ss.).

3 Indicaremos los precursores de nuestra interpretación en la Sección II. 
rías y la multiplicidad empírica parece tornar impracticable la aplicación de las primeras a la segunda.

Dicho en pocas palabras, la heterogeneidad entre las categorías y la multiplicidad empírica consiste en que al contenido de las primeras, a saber, lo que es pensado en ellas, no le corresponden elementos sensibles. Por un lado, el contenido de las categorías proviene enteramente del entendimiento y es completamente independiente de la intuición espacio-temporal humana. Por este motivo, las categorías no son conceptos limitados a los objetos empíricos dados a nuestra intuición, sino que permiten pensar objetos de una intuición en general no especificada. Por otro lado, la sensibilidad recibe la multiplicidad empírica, sin intervención del entendimiento, cuando es afectada por un ente independiente de la mente. Por lo tanto, la multiplicidad empírica es un factor meramente dado que no presenta forma intelectual alguna, sino únicamente las formas de la sensibilidad, esto es, el espacio y el tiempo.

La aplicación de un concepto a una multiplicidad intuitiva es posible cuando en la segunda pueden percibirse los elementos sensibles correspondientes a los elementos intelectuales pensados en ese concepto. Sin embargo, hemos visto que el contenido puramente intelectual de las categorías no tiene equivalente en la multiplicidad empírica. Por tanto, la aplicación de las categorías no es posible inmediatamente. Tal aplicación presupone la mediación de los esquemas trascendentales. Los esquemas trascendentales expresan el contenido de las categorías en términos de la temporalidad de la experiencia posible y de esa manera hacen posible que aquél se vincule con la multiplicidad empírica.

Cabe destacar que nuestra exposición presupone una interpretación de la naturaleza de los esquemas trascendentales que hemos desarrollado en otro lugar. ${ }^{4}$ Consideramos que los esquemas trascendentales son procedimientos de síntesis de la imaginación y al mismo tiempo las propiedades temporales fundamentales de los objetos empíricos producidas por semejantes procedimientos. Llamaremos a estos aspectos de los esquemas trascendentales, respectivamente, esquemas-procedimientos y esquemas-productos. La multiplicidad empírica se constituye como objeto empírico cuando, en virtud de las síntesis de los esquemas-procedimientos, presenta las propiedades temporales fundamentales o esquemas-productos. En la sección II veremos que esos esquemas-productos, exceptuando los correspondientes a las categorías de la modalidad, pueden entenderse como las propiedades ontológicas fundamentales que todo objeto empírico tiene que presentar para ser tal. ${ }^{5}$

4 Cf. M.Arias Albisu, «Los esquemas trascendentales como procedimientos y productos», Revista de filosofía (Madrid), 35, 2 (2010), pp. 27-42.

5 Los esquemas correspondientes a las categorías de la cantidad y la cualidad son presentados en el «Capítulo del esquematismo» como esquemas-procedimientos (no obstante, notemos 


\section{CATEGORÍAS, ESQUEMAS Y PRINCIPIOS}

Como hemos adelantado, los esquemas trascendentales posibilitan la subsunción de los fenómenos bajo las categorías, esto es, la subsunción trascendental. ${ }^{6}$ Esta subsunción es expresada en un juicio categórico cuyo sujeto es un aspecto del orden fenoménico y su predicado una categoría. Kant expone y pretende demostrar estos juicios en el «Sistema de los principios». El objetivo de la presente sección es esclarecer la relación entre las categorías, los esquemas trascendentales y esos principios del entendimiento. Mostraremos que los principios del entendimiento ponen en contacto las categorías y los fenómenos tan sólo mediante los esquemas trascendentales.

El último párrafo de la exposición general de los principios del entendimiento correspondientes a las categorías de la relación, a saber, las «Analogías de la experiencia», trata las relaciones entre principios, categorías y esquemas. Lo citamos a continuación.

Pero lo que se había advertido con respecto a todos los principios sintéticos, y que aquí debe ser notado especialmente, es esto: [...] que en consecuencia, los fenómenos no deben ser subsumidos directamente bajo las categorías, sino solamente bajo los esquemas de ellas. [...] Por consiguiente, mediante estos principios

que la referencia al esquema como «procedimiento» del «Capítulo del esquematismo» es válida únicamente para los esquemas de los conceptos empíricos y matemáticos - cf. A 140/B 179-. Sin embargo, en Crítica de la razón práctica Kant se refiere al esquema trascendental como a un «procedimiento universal de la imaginación»; AA, V, 69). Las propiedades producidas por esos procedimientos (esquemas-productos) se estudian en las secciones dedicadas a los principios del entendimiento correspondientes a esas categorías ( «Axiomas de la intuición» y «Anticipaciones de la percepción»). En cambio, los esquemas de las categorías de la relación y la modalidad son presentados como propiedades o esquemas-productos ( $c f$. A 144-145/B 183-184. La perspectiva del esquema como producto se pone en evidencia asimismo cuando Kant trata el esquema trascendental como «un tercero», «representación mediadora»-A 138/B 177- y «un producto trascendental de la imaginación»-A 142/B 181-). Queda a cargo del intérprete la reconstrucción de los procedimientos de síntesis que dan lugar a esas propiedades. Para una reconstrucción del esquema-procedimiento de la categoría de substancia y accidente, $c f$. A. Rosales: «Una pregunta sobre el tiempo», en A. Rosales: Siete ensayos sobre Kant. Mérida: Universidad de los Andes, 1993, pp. 225-250, aquí pp. 237-239. Tal vez sea conveniente ofrecer un ejemplo. El esquemaprocedimiento de la cualidad es una intensificación progresiva a partir de la ausencia de cualidad, o una disminución sucesiva de la intensidad de una cualidad ( $c f$. A 143/B 182-183). Por su parte, el esquema-producto de la cualidad es el grado o magnitud intensiva ( $c f$. B 207-208). La síntesis del esquema-procedimiento determina las cualidades del fenómeno como cualidades con un cierto grado de intensidad, es decir, cualidades de objetos empíricos constituidos como tales. Nos limitamos a señalar la plausibilidad de esta interpretación, puesto que la fundamentación de la misma requiere un estudio independiente.

6 Carta a Tieftrunk, 11 de diciembre de 1797 (AA, XII, 224). 
estaremos facultados para componer, sólo según una analogía, los fenómenos con la unidad lógica y universal de los conceptos; y por eso, en el principio mismo nos serviremos, por cierto, de la categoría, pero en la ejecución (en la aplicación a fenómenos) pondremos en su lugar a su esquema, como clave del uso de ella; o más bien, se lo pondremos a aquélla al lado, como condición restrictiva, con el nombre de una fórmula del primero. ${ }^{7}$

Al comienzo del pasaje citado se establece que las tesis que serán expuestas en lo que sigue habían sido sostenidas con respecto a todos los principios. Tal afirmación implica, en primer lugar, que lo que sigue no es válido únicamente respecto de las analogías de la experiencia, sino que es aplicable a todos los principios del entendimiento. En segundo lugar, la afirmación mencionada señala que lo que se ha de exponer no introduce ninguna novedad substancial con respecto a lo explicado hasta el momento.

Los fenómenos, afirma Kant, no deben ser subsumidos directamente bajo las categorías, sino únicamente bajo sus esquemas. Esta proposición presenta dificultades, dado que el esquema es una condición para la subsunción bajo conceptos, y no un concepto bajo el cual podrían subsumirse fenómenos. Es cierto que los fenómenos no pueden ser subsumidos directamente bajo las categorías, en virtud de la heterogeneidad que se presenta entre los términos. Sin embargo, el esquema es introducido como mediador para resolver el problema de la heterogeneidad, de modo que la subsunción tenga lugar, si bien mediatamente, bajo las categorías.

Al final del texto Kant se expresa de un modo un tanto más preciso. En el principio del entendimiento mismo, afirma Kant, nos servimos de la categoría. Consideramos que esta tesis debe entenderse en el sentido de que la subsunción tiene lugar en última instancia bajo la categoría. En la aplicación a los fenómenos, continúa Kant, pondremos el esquema en lugar de la categoría como clave de su uso; o, más bien, al lado de ella, como condición restrictiva, ${ }^{8}$ con el nombre de fórmula de su uso. El esquema sería la clave o fórmula del uso de la categoría en el ámbito fenoménico. Procuraremos interpretar esta afirmación. Las categorías rigen una síntesis de la multiplicidad de una intuición en general. Ahora bien, la única intuición que es dada al ser humano es la espacio-temporal. A fin de constituir un objeto empírico, las categorías deben dirigirse a tal intuición. Por consiguiente, las categorías tienen que unificar los fenómenos espacio-temporales. Semejante unificación tiene lugar mediante la

7 A 180-181/B 223-224.

8 La expresión «condición restrictiva» hace referencia al hecho de que la posibilitación del empleo de las categorías es al mismo tiempo una restricción de su ámbito de uso objetivo. Los esquemas trascendentales posibilitan la subsunción de los fenómenos bajo las categorías, y precisamente por ello limitan su campo de aplicación al ámbito fenoménico. 
síntesis de la imaginación. Los esquemas trascendentales son precisamente procedimientos y productos de tal síntesis. El esquema es clave del uso de la categoría en su aplicación a los fenómenos en la medida en que representa la especificación de la síntesis de una multiplicidad en general en una síntesis de una multiplicidad espacio-temporal, y en que esta especificación hace posible el empleo de las categorías en el ámbito fenoménico.

Puede afirmarse que el pasaje considerado no aporta precisiones ulteriores acerca del problema de la subsunción trascendental tal como éste es tratado en el «Capítulo del esquematismo». La formulación de un principio no presupone la subsunción de la multiplicidad empírica bajo los esquemas, sino, como ya habíamos anticipado, bajo las categorías por intermedio de los esquemas.

Intentaremos mostrar cómo se relacionan las categorías, los esquemas y los principios. De acuerdo con la interpretación de los esquemas trascendentales esbozada en la introducción de este trabajo, la subsunción trascendental presupone la producción de propiedades temporales fundamentales, a saber, esquemas-productos. Consideramos que en los principios del entendimiento una categoría es atribuida como predicado a un aspecto del ámbito fenoménico. Esta atribución presupone la imposición de los esquemas-productos, correspondientes a cada categoría, a los respectivos aspectos del ámbito fenoménico. Los principios del entendimiento expresan la subsunción de la multiplicidad empírica bajo las categorías y, por tanto, implican la síntesis de toda multiplicidad empírica por parte de los esquemas-procedimientos, de modo tal que esta multiplicidad presente las propiedades temporales fundamentales o esquemas-productos. La multiplicidad empírica se constituye como objeto empírico cuando presenta tales propiedades. ${ }^{9}$

A fin de aclarar esta cuestión, tal vez sea conveniente dar un ejemplo. Consideremos el principio que se deriva de la aplicación de la categoría de causalidad y dependencia. Según la primera edición de $K r V$, la segunda analogía de la experiencia establece: «Todo lo que ocurre (comienza a ser) presupone algo a lo cual sigue según una regla». ${ }^{10}$ A un aspecto del ámbito fenoménico, a saber, la alteración, le es atribuido como propiedad temporal fundamental el esquema de la categoría de causalidad y dependencia. Tal esquema, precisamente, consiste «[...] en la sucesión de los múltiple, en la medida en que está

9 Véase la presentación de la relación entre categorías, esquemas y principios efectuada por Peter Krausser. Cf. P. Krausser, «Zu einer systematischen Rekonstruktion der Erkenntnis und Wissenschaftstheorie in Kants Kritik der reinen Vernunft. Teil VI: Zum Verhältnis von Kategorien, Schemata und Grundsätzen und der Rolle der Analogien-Kapitel in der sogenannten 'Deduktion'», Kant Studien, 67, 2 (1976), pp. 141-155, aquí p. 143.

10 A 189. 
sometida a una regla». ${ }^{11}$ Según la segunda edición de $K r V$, la segunda analogía establece: «Todas las alteraciones suceden según la ley de la conexión de la causa y el efecto». ${ }^{12}$ En esta formulación le es atribuida a un aspecto del ámbito fenoménico (a saber, la alteración) la categoría de causalidad y dependencia. Consideramos que esta diferencia entre las formulaciones de 1781 y 1787 no implica un cambio de perspectiva, sino que se debe a que la subsunción trascendental, por un lado, tiene lugar bajo las categorías y, por el otro, presupone la imposición de esquemas-productos.

Antes de concluir la presente sección, nos ocuparemos de un problema adicional que presenta el pasaje de las «Analogías de la experiencia» citado más arriba. Kant afirma que mediante los principios componemos (zusammensetzen), aunque sólo según una analogía (Analogie), los fenómenos con la unidad lógica y universal de los conceptos. Inmediatamente a continuación se establece que el esquema es clave del uso de la categoría en su aplicación a los fenómenos. Hemos mostrado, por un lado, que un principio del entendimiento es un juicio cuyo predicado es una categoría y, por el otro, que tal juicio presupone la imposición del esquema-producto, correspondiente a la categoría, a un aspecto del ámbito fenoménico. La categoría y los fenómenos se vinculan en el principio del entendimiento por intermedio del esquema trascendental. Por consiguiente, la composición entre fenómenos y contenido lógico de las categorías tiene lugar, a decir verdad, mediante los esquemas trascendentales.

Consideramos que la composición mencionada se realiza según una analogía en virtud de que los esquemas-procedimientos generan esquemas-productos que son análogos temporales de las categorías. En efecto, los esquemas-procedimientos, regidos por lo pensado en las categorías, generan en la multiplicidad empírica propiedades temporales o esquemas-productos. ${ }^{13}$ En los esquemas-productos el

11 A 144/B 183.

12 B 232.

13 Podría objetársenos que el esquema trascendental no consiste en una propiedad de la multiplicidad empírica, sino en una propiedad del tiempo puro. De hecho, Kant define el esquema trascendental como "transscendentale Zeitbestimmung" (A 138/B 177). Esta expresión es traducida habitualmente por "determinación trascendental del tiempo". Sin embargo, la expresión mencionada también puede ser traducida por "determinación trascendental temporal". Según esta interpretación, el esquema no sería una determinación del tiempo puro, sino una determinación temporal de intuiciones empíricas espacio-temporales. Tanto Paton como Allison y Düsing han afirmado que el esquema trascendental no consiste en una determinación del tiempo puro, sino en una determinación temporal de lo empírico. Cf. H. J. Paton, Kant's Metaphysic of Experience. London: George Allen \& Unwin Ltd., 1970, t. II, pp. 28-30; H. E. Allison, El idealismo trascendental de Kant: una interpretación y defensa, tr. D. M. Granja Castro. Barcelona: Anthropos, 1992, pp. 286-287; y K. Düsing, «Schema und Einbildungskraft in Kants Kritik der reinen Vernunft», en L. Kreimendahl (ed.), Aufklärung und Skepsis. Studien zur Philosophie und 
contenido de las categorías y la multiplicidad empírica en su dimensión temporal están compuestos o enlazados. Un esquema-producto puede ser comprendido entonces como un análogo temporal de una categoría.

Señalemos que «analogía» no tiene en el pasaje examinado el sentido que Kant concede al término en la expresión «analogía de la experiencia». A fin de demostrar esta última tesis, expondremos brevemente el sentido de «analogía» que es privativo de las analogías de la experiencia. En las «Analogías de la experiencia» Kant distingue entre una analogía matemática y una filosófica. La analogía matemática, o proporción, es una fórmula que expresa la igualdad de dos relaciones cuantitativas. Por ejemplo, 4 es a 2 como 16 es a 8 . Kant señala que esta relación es constitutiva, puesto que si están dados tres términos es posible determinar a priori el cuarto. Las analogías de la filosofía son propiamente las analogías de la experiencia. Tales analogías expresan la igualdad de dos relaciones cualitativas. En este caso la relación no es constitutiva, sino regulativa. Si están dados tres términos, podemos de hecho conocer a priori la relación con el cuarto. Pero no es posible determinar a priori cuál sea propiamente el cuarto miembro. Lo único que poseemos, afirma Kant, es una regla para buscar el cuarto término en la experiencia y una señal para encontrarlo en ella. Por ejemplo, en el caso de la categoría de causalidad y dependencia, se puede afirmar que el efecto es a la causa como el derretimiento de la cera es a $x$. Ciertamente, no podemos conocer a priori cuál es el estado que ocasionó el derretimiento de la cera. Con todo, sabemos que debemos buscar en la experiencia un estado del cual el derretimiento de la cera se siga necesariamente en el tiempo y según una regla.

Es evidente que el sentido de «analogía» que acabamos de exponer es privativo de las analogías de la experiencia, y que el sentido de «analogía» que es válido respecto de todos los principios del entendimiento es diferente del primero. En efecto, cuando afirmamos que un esquema-producto es un análogo temporal de una categoría no aludimos con ello a ningún cuarto término desconocido. Más bien, indicamos que los esquemas-procedimientos expresan en propiedades temporales o esquemas-productos el contenido puramente lógico e intelectual de las categorías. En conclusión, los esquemas-productos son aná-

Geistesgeschichte des 17. und 18. Jahrhunderts. Günter Gawlick zum 65. Geburtstag. StuttgartBad Cannstatt: Frommann-Holzboog, 1995, pp. 47-71, aquí p. 69. Debemos el señalamiento de esta ambigüedad en la definición kantiana de los esquemas trascendentales a Mario Caimi. Caimi traduce 'transscendentale Zeitbestimmung' por 'determinación trascendental del tiempo' y da noticia de la traducción alternativa preferida por nosotros en p. 244, n. 525 de su edición de la Crítica de la razón pura. 
logos temporales de las categorías en la medida en que expresan o interpretan temporalmente el contenido de las mismas. ${ }^{14}$

\section{EL «SISTEMA DE LOS PRINCIPIOS» COMO FUNDAMENTO DE LA ONTOLOGÍA CRÍTICA KANTIANA}

El objetivo de esta sección es defender la tesis según la cual los esquemasproductos, exceptuando los correspondientes a las categorías de la modalidad, ${ }^{15}$ son las propiedades ontológicas fundamentales de los objetos empíricos A fin de justificar esta afirmación, demostraremos que el «Sistema de los principios» puede ser interpretado como un tratado de ontología.

Kant afirma que los principios a priori del entendimiento llevan ese nombre porque contienen el fundamento de otros juicios y no están basados en conocimientos superiores ni más generales. ${ }^{16}$ Los principios del entendimiento estatuyen la posibilidad de la experiencia. Establecen las condiciones que debe satisfacer un objeto a fin de ser un objeto de experiencia posible. ${ }^{17}$ Por consiguiente, los principios no se derivan de ningún conocimiento empírico particular ni representan generalizaciones de conocimientos empíricos particulares. Por el contrario, todo juicio acerca de un objeto o ámbito objetivo particular presupone los principios del entendimiento.

A continuación intentaremos mostrar que las proposiciones fundamentales establecidas en el «Sistema de los principios» pueden caracterizarse como ontológicas. La tarea, a primera vista, parece no presentar dificultades. En efecto, hemos establecido que los principios del entendimiento determinan en su integridad el ser del objeto empírico en general. Sin embargo, demostrar que Kant mismo comprendió el «Sistema de los principios» como una ontología exige un análisis de los textos en los que se pronunció sobre esa disciplina metafísica. Nos ocuparemos en lo que sigue de presentar los pasajes más relevantes para nuestros fines.

El primer texto es citado a menudo por comentaristas y parece contradecir nuestra hipótesis. En efecto, una lectura rápida del mismo podría llevarnos a

14 Cf. A 179-180/B 221-223. Cf. H. J. Paton, op. cit., t. II, pp. 179-183; y H. E. Allison, op. cit., pp. 304-307.

15 Las categorías de la modalidad no determinan, mediante las síntesis de sus esquemasprocedimientos, propiedades de los objetos empíricos considerados en sí mismos, sino que establecen únicamente relaciones entre los objetos empíricos constituidos como tales y la facultad cognoscitiva en su uso empírico. $C f$. A 219/B 266.

$16 C f$. A 148/B 188.

17 En efecto, las condiciones de posibilidad de la experiencia en general son al mismo tiempo condiciones de posibilidad de los objetos de la experiencia. Cf. A 158/B 197. 
pensar que Kant consideraba que la filosofía crítica no admite reflexiones de orden ontológico.

De aquí se sigue innegablemente: que los conceptos puros del entendimiento nunca pueden ser de uso transcendental, sino que pueden ser siempre sólo de uso empírico, y que los principios del entendimiento puro sólo pueden, en relación con las condiciones generales de una experiencia posible, ser referidos a objetos de los sentidos, y nunca a cosas en general (sin tener en cuenta la manera como las intuyamos).

La analítica transcendental tiene, según esto, este importante resultado: que el entendimiento no puede nunca hacer, a priori, más que anticipar la forma de una experiencia posible en general; y que, puesto que aquello que no es fenómeno no puede ser objeto de la experiencia, [el entendimiento] no puede nunca sobrepasar las limitaciones de la sensibilidad, sólo dentro de las cuales nos son dados objetos. Los principios de él son meros principios de la exposición de los fenómenos, y el orgulloso nombre de una ontología que pretende suministrar, en una doctrina sistemática, conocimientos sintéticos a priori de cosas en general (p. ej. el principio de causalidad), debe dejar su lugar al más modesto de una mera analítica del entendimiento puro. ${ }^{18}$

En más de una ocasión se establece en el pasaje citado que los principios del entendimiento sólo son válidos para los objetos de una experiencia posible. El entendimiento anticipa la forma de los objetos susceptibles de darse a una intuición espacio-temporal humana. Esta circunstancia se debe a que las categorías sólo pueden ser empleadas en los principios una vez que se les han asignado esquemas. En efecto, los esquemas restringen el campo de aplicabilidad de las categorías a la multiplicidad espacio-temporal. Por consiguiente, los principios del entendimiento no son válidos respecto de las cosas en sí mismas, es decir, consideradas independientemente de su relación con la sensibilidad humana.

Al final del pasaje se afirma que la ontología debe dejar su lugar a una analítica del entendimiento puro. Ahora bien, la ontología se entiende allí como una doctrina sistemática que proporciona conocimientos sintéticos a priori de las cosas en general. Puede interpretarse entonces que Kant no rechaza toda forma de ontología, sino lo que podría llamarse ontología dogmática. La ontología dogmática pretende proporcionar conocimientos sistemáticos de las cosas en sí mismas sin efectuar un previo examen crítico del ámbito de validez de los conceptos y principios de la razón humana. ${ }^{19}$ Queda entonces abierta la posibilidad de considerar el «Sistema de los principios» como un tratado de ontología no dogmática. Tal ontología podría denominarse crítica. 
Kant vuelve a tratar la problemática de la ontología en la «Arquitectónica de la razón pura». Esta sección de $K r V$ toma por objeto la forma sistemática del conocimiento. El conocimiento racional es o bien filosófico, o bien matemático. El conocimiento racional filosófico es o bien un conocimiento por razón pura, o un conocimiento racional por principios empíricos. El primer conocimiento se llama filosofía pura, mientras que el segundo, filosofía empírica.

La filosofía pura posee una parte propedéutica, a saber, la crítica, y una parte sistemática llamada metafísica. La crítica investiga la facultad de la razón con respecto a todos los conocimientos a priori. La metafísica, a su vez, se considera como el conocimiento filosófico por razón pura en su interconexión sistemática. ${ }^{20}$ Tiene la metafísica una parte especulativa y una parte práctica. La primera se denomina metafísica de la naturaleza; la segunda, metafísica de las costumbres. Por su parte, la metafísica de la naturaleza se divide en filosofía trascendental y fisiología de la razón pura. Reproducimos a continuación el pasaje en el que Kant describe estas ramas de la metafísica especulativa.

La primera [MAA: filosofía trascendental] estudia sólo el entendimiento y la razón misma en un sistema de todos los conceptos y principios que se refieren a objetos en general, sin suponer objetos que estuvieran dados (ontología); la segunda [MAA: fisiología de la razón pura] estudia la naturaleza, es decir, el conjunto de los objetos dados (ya sean dados a los sentidos, o si se quiere, a alguna otra especie de intuición), y es, por consiguiente, una fisiología (aunque solo rationalis). ${ }^{21}$

En el pasaje citado la filosofía trascendental u ontología se concibe como el sistema de todos los conceptos y principios que se refieren a objetos en general, sin suponer objetos que estuvieran dados. Es posible entender tal descripción de dos maneras diferentes. En primer lugar, puede suponerse que la ontología versa sobre los conceptos y principios que se refieren a cosas en sí mismas. Ahora bien, no se comprendería entonces la equiparación entre esta ontología concebida de manera dogmática y la filosofía trascendental. En segundo lugar, puede interpretarse que la ontología versa sobre los principios que se refieren a objetos de la experiencia en general, sin atender a objetos determinados o particulares de la experiencia. En este caso, el «Sistema de los principios» formaría parte de la ontología. Afirmamos que el «Sistema de los principios» es sólo una parte de la filosofía trascendental u ontología, y no el sistema completo de la misma, en virtud de que aquél es expuesto en la crítica de la razón. En efecto, la crítica de la razón, si bien se ocupa de la totalidad de los conceptos

20 En este contexto, «razón» (Vernunft) debe ser entendido en un sentido amplio. Designa el conjunto de las facultades superiores del conocimiento (entendimiento, facultad de juzgar y razón en sentido estricto) y sirve para contrastar lo racional y lo empírico. $C f$. A 11/B 25, A 13/B 27, A $835 / \mathrm{B} 863$.

21 A $845 / \mathrm{B} 873$. 
primitivos del conocimiento a priori, se abstiene de un análisis detallado de los mismos y de la reseña completa de los conceptos que se derivan de ellos. ${ }^{22}$ Por consiguiente, la crítica de la razón presenta únicamente los fundamentos del sistema de la ontología.

Nuestra interpretación según la cual el «Sistema de los principios» forma parte de la ontología es confirmada por lo que se dice a continuación. ${ }^{23}$ La fisiología racional trata la naturaleza como conjunto de objetos dados. Se divide en fisiología inmanente y fisiología trascendente. La fisiología trascendente se refiere a conexiones de los objetos de la experiencia que rebasan los límites de la experiencia posible. Por tanto, su consideración no es relevante para nuestros fines. La fisiología inmanente, en cambio, se ocupa de la naturaleza como conjunto de los objetos dados a nuestros sentidos y toma en consideración las distantes clases de objetos. Se divide en física racional y psicología racional. Es evidente que la fisiología inmanente presupone clases de objetos de una naturaleza o constitución determinada, a saber, cuerpos materiales (naturaleza corpórea) y seres pensantes (naturaleza pensante). Por este motivo Kant ha diferenciado la fisiología inmanente de la ontología. ${ }^{24}$ Cuando Kant afirma, entonces, que la ontología estudia «objetos en general» no tiene in mente las cosas en sí mismas, sino la totalidad de los objetos de la experiencia posible y sin atender a su constitución específica ni a conexiones de ellos que sobrepasan los límites de la experiencia.

Consideramos que el pasaje citado aporta evidencia textual suficiente para interpretar el «Sistema de los principios» como un tratado de ontología. A continuación nos ocuparemos de dos textos que arrojan resultados similares. En primer lugar, en la Crítica de la facultad de juzgar las categorías son denominadas predicados ontológicos. ${ }^{25}$ Ahora bien, las categorías son los predicados de los juicios sintéticos a priori en los que consisten los principios del entendimiento. Si tales predicados son ontológicos, entonces también lo son los principios correspondientes. En segundo lugar, del siguiente texto de Los progresos de la metafísica se desprende que el «Sistema de los principios» es parte integrante de la ontología.

La ontología es aquella ciencia (como parte de la metafísica) que consiste en un sistema de todos los conceptos y principios del entendimiento, pero sólo en cuanto se refieren a objetos que pueden ser dados por los sentidos y que por consiguiente

22 Cf. A 13-14/B 27-28 y A XXI.

23 Cf. A 845-846/B 873-874.

24 Cf. Primeros principios metafísicos de la ciencia de la naturaleza, «Prefacio» (AA, IV, 469-470).

25 «Predicados ontológicos (conceptos puros del entendimiento).» Crítica de la facultad de juzgar, «Introducción», V (AA, V, 181). 
pueden ser documentados por la experiencia. No toca lo suprasensible, lo cual es, empero, el fin último de la metafísica, y por consiguiente pertenece a ésta sólo como propedéutica, como la antesala o el atrio de la metafísica propiamente dicha, y se la llama filosofía transcendental porque contiene las condiciones y los primeros elementos de todo nuestro conocimiento a priori ${ }^{26}$

La ontología es considerada aquí como una parte de la metafísica. ${ }^{27}$ Consiste en el sistema de todos los principios del entendimiento que se refieren a objetos de los sentidos. Se indica que tal ontología no alcanza lo suprasensible, es decir, no es trascendente. Debe concebírsela por tanto como inmanente. ${ }^{28} \mathrm{Al}$ final del pasaje se equipara ontología con filosofía trascendental.

Hemos demostrado que el «Sistema de los principios» puede entenderse como un tratado de ontología. Los principios del entendimiento, entonces, hacen posible la verdad trascendental. En efecto, los principios son conocimientos que determinan el ser de los objetos empíricos. Y la verdad trascendental consiste en la necesaria concordancia de la objetividad de los objetos empíricos con los principios ontológicos del entendimiento. La verdad trascendental es lógicamente anterior a la verdad empírica y la hace posible, pues todo conocimiento empírico verdadero presupone la concordancia del objeto conocido con los principios del entendimiento. ${ }^{29}$

Si un principio ontológico del entendimiento presupone la imposición de un esquema-producto, correspondiente a una categoría, a un aspecto de la multiplicidad empírica, entonces los esquemas-productos son las propiedades ontológicas fundamentales. ${ }^{30}$ Por tanto, puede afirmarse que la doctrina del

26 Los progresos de la metafísica (AA, XX, 260). Cf. también AA, XX, 281, 286, 315316.

27 Para un análisis del concepto de metafísica expuesto en Los progresos de la metafísica, cf. M. Caimi, La metafísica de Kant. Reconstrucción de la argumentación del escrito de Kant «Los progresos de la metafísica desde la época de Leibniz y de Wolff». Buenos Aires: EUDEBA, 1989.

28 En la carta a Beck de 20 de enero de 1792 Kant indica que ha hecho un bosquejo de un sistema de metafísica. Comenzaría la primera sección con una exposición de las categorías según su orden. Mostraría después que no sería posible ninguna experiencia de objetos de los sentidos si ellos no fueran pensados mediante las categorías y no fueran representados como dados en el espacio y en el tiempo. Kant afirma a continuación: «De allí surge una ciencia completa de la ontología como pensamiento inmanente, esto es, [MAA: una ciencia] de aquél [MAA: pensamiento] en el cual se puede asegurar a conceptos su realidad objetiva.» AA, XI, 314. Longuenesse cita y comenta este pasaje. $C f$. B. Longuenesse, Kant and the Capacity to Judge, tr. Ch. T. Wolfe. Princeton: Princeton University Press, 2000, pp. 394-399.

29 Cf. A 146/B 185, A 237/B 296. Para un análisis del problema, $c f$. Ch.-H. Paek, «Kant's Theory of Transcendental Truth as Ontology», Kant Studien, 96, 2 (2005), pp. 147-160.

30 Alberto Rosales ha sostenido que los esquemas trascendentales son las determinaciones 
esquematismo trascendental desempeña un papel principal en la posibilitación de la ontología crítica. En efecto, tan sólo mediante el esquematismo trascendental son producidas las propiedades ontológicas fundamentales, es decir, los esquemas-productos. ${ }^{31}$

ontológicas concretas del objeto. «En efecto, las categorías por un lado, o las intuiciones puras de espacio y tiempo, por el otro, son en cada caso sólo partes de la determinación ontológica del objeto. Ambos momentos se encuentran reunidos en cambio por primera vez en los esquemas trascendentales, que son de tal modo las determinaciones ontológicas concretas del objeto». A. Rosales, op.cit., p. 229.

31 Una lectura de $\mathrm{KrV}$ en términos de ontología ha sido defendida por Martin Heidegger. Heidegger considera que $K r V$ trata la ontología como un problema, es decir, se plantea el problema de la posibilidad interna de la ontología. Cf. M. Heidegger, Kant und das Problem der Metaphysik. Frankfurt am Main: Vittorio Klostermann, 1991, pp. 5-19. La sección dedicada al esquematismo trascendental es denominada por Heidegger «El fundamento de la posibilidad interna del conocimiento ontológico». Coincidimos con Heidegger cuando sostiene que mediante el esquematismo trascendental se constituye el horizonte de comprensión ontológica dentro del cual se ofrecen los entes. Ibid., pp. 101-108. (Ahora bien, ciertas tesis de Heidegger dan a entender que su valoración e interpretación del esquematismo trascendental vienen determinadas por su problemática caracterización de la imaginación como raíz común en la que se originan entendimiento y sensibilidad. «Si Kant plantea en el capítulo del esquematismo el problema de la conceptualidad de los conceptos originarios y lo resuelve con ayuda de la determinación de la esencia de estos conceptos como esquemas trascendentales, entonces la doctrina del esquematismo de los conceptos puros del entendimiento es el estadio decisivo de la fundamentación de la metaphysica generalis». Ibid., p. 111. La afirmación de que la esencia de las categorías viene dada por los esquemas trascendentales parecería indicar que las categorías mismas se originan en los esquemas trascendentales en tanto productos trascendentales de la imaginación. Ibid., pp. 146-155. Efectivamente, notemos que Hermann Mörchen, quien sigue en lo esencial a Heidegger en su concepción de la imaginación, ha sostenido que el concepto y las imágenes de la percepción son elementos pertenecientes a la estructura conformada por el esquema. $C f$. H. Mörchen, Die Einbildungskraft bei Kant. Tübingen: Max Niemeyer, 1970, p. 118. Aquí, en cambio, consideramos que la imaginación es el entendimiento mismo cuando atiende no ya solamente a las leyes de la lógica, sino también a las peculiaridades de nuestra intuición sensible. Para un desarrollo de esta concepción, $c f$. M. Caimi, «Comments on the conception of Imagination in the Critique of Pure Reason», en Akten des X. Internationalen Kant-Kongresses. Band 1: Hauptvorträge. Berlín-New York: Walter de Gruyter, 2008, pp. 39-50). Por su parte, Herbert J. Paton estima que la doctrina del esquematismo es un momento necesario de lo que denomina «metafísica de la experiencia». Cf. H. J. Paton, op. cit., t. I, p. 22 y t. II, pp. 17-20. Según Henry E. Allison, se puede aceptar la interpretación de Heidegger siempre y cuando se entienda, como Paton, la metafísica de una manera kantiana. La metafísica así concebida sería un conjunto de proposiciones sintéticas a priori que expresan la aplicación de las categorías al ámbito de los fenómenos. $C f$. H. E. Allison, op. cit., p. 275. 


\section{UNA CONCEPCIÓN ALTERNATIVA: LA INTERPRETACIÓN SEMÁNTICA DEL ESQUEMA- TISMO TRASCENDENTAL. PRESENTACIÓN Y CRÍTICA.}

La última sección del presente artículo está dedicada a la discusión de un grupo de interpretaciones de la doctrina del esquematismo. ${ }^{32}$ Lo distintivo de estas lecturas es que consideran que el esquematismo trascendental proporciona una interpretación de las categorías. «Interpretación» está tomado aquí aproximadamente en el sentido que tiene el término en la semántica lógica. Dicho en otras palabras, las categorías serían, si bien no símbolos de un lenguaje formal, entidades formales a las cuales el esquematismo trascendental asociaría con elementos objetivos de un conjunto denominado dominio de interpretación. A través de la crítica de este grupo de interpretaciones mostraremos de manera indirecta la corrección de nuestra interpretación ontológica del esquematismo trascendental.

Expondremos en primer lugar las tesis de Robert E. Butts. En segundo lugar, presentaremos los lineamientos generales de la lectura de Zeljko Loparic. Por último, haremos algunas observaciones críticas con respecto a las interpretaciones semánticas del esquematismo.

Butts considera que las categorías proporcionan una gramática epistemológica (y no meramente lógica) para todo sistema que pretenda formular proposiciones verdaderas acerca de cuestiones de hecho. Por ejemplo, la categoría epistemológica de causalidad denota un componente gramatical de ciertos juicios en el mismo sentido en que la categoría sintáctica de sustantivo denota componentes gramaticales de algunas oraciones del español. Ahora bien, una gramática, aunque sea epistemológica, necesita ser complementada por reglas de formación que hagan posibles juicios o proposiciones significativas. Butts considera que, para Kant, este papel es desempeñado en gran parte por el «Sistema de los principios». Allí no se trataría tanto de hacer posible el conocimiento inmediato, sino de determinar las condiciones a priori de las generalizaciones empíricas que puedan llegar a ser leyes de la naturaleza. Los principios del entendimiento especificarían de modo a priori la forma de las leyes de la naturaleza. El principio causal, por ejemplo, determina la forma de todas las leyes que describen eventos causales.

Según Butts, las categorías y los principios proporcionan únicamente reglas para formar un sistema conceptual de un cierto tipo. Corresponde a la experiencia proveer los contenidos específicos. Dicho en otras palabras, el sistema kantiano de las categorías y las reglas de construcción a priori es un

32 Nos referimos a R. E. Butts, «Kant's Schemata as Semantical Rules», en L. W. Beck (ed.), Kant Studies Today. La Salle: Open Court, 1969, pp. 290-300; y a Z. Loparic, A semântica transcendental de Kant. Campinas: UNICAMP, 2000. 
formalismo epistémico no interpretado. El esquematismo es introducido precisamente para dar respuesta a la pregunta por la aplicación de este formalismo a las instancias empíricas. De hecho, los esquemas trascendentales pueden ser entendidos como reglas semánticas que vinculan las categorías con predicados observacionales. Los esquemas especifican de manera general qué clases de predicados observacionales son admisibles en función de la forma epistémica del sistema. Por ejemplo, el esquema de las categorías de la cualidad legitima la clase de los predicados que adscriben grados específicos de intensidad a los objetos empíricos. ${ }^{33}$ Es decir, predicados observacionales como « $10^{\circ} \mathrm{C}$ s serán tenidos por válidos.

Butts estima que los esquemas trascendentales determinan el contenido formal de los predicados observacionales que satisfacen los otros requisitos formales del sistema. Utiliza la expresión «contenido formal» para indicar que los esquemas no nos dicen qué serán los detalles de la experiencia en tanto detalles, sino que ellos nos dicen qué clases de detalles deben ser consideradas en la empresa científica. Dicho en otras palabras, los esquemas proporcionan la forma más general de los predicados observacionales. ${ }^{34}$

Por su parte, Loparic introduce el término «semántica trascendental» para designar la parte de la lógica trascendental que estudia cómo ciertas representaciones son aplicadas a priori. ${ }^{35}$ Así como Butts afirma que las categorías constituyen una gramática epistemológica y no meramente lógica, Loparic estima que su concepción de la semántica no es idéntica a la de la lógica contemporánea. Los conceptos de que habla Kant, en efecto, no son elementos de un lenguaje formal, ni siquiera de un lenguaje natural, sino representaciones mentales. ${ }^{36}$

33 Cf. R. E. Butts, op. cit., pp. 290-294.

34 Cf. Ibid., pp. 297-298. Puede afirmarse que el trabajo de Stephan Körner inició la corriente de interpretación del esquematismo que desarrolla Butts. Körner distingue entre, por un lado, la gramática lógica de los conceptos, a saber, las reglas no referenciales que rigen el empleo de los conceptos cuando ellos se relacionan con otros conceptos, y, por el otro, las reglas referenciales (esquemas) que relacionan los conceptos con los objetos percibidos. $C f$. S. Körner, Kant, tr. I. Zapata Tellechea. Madrid: Alianza, 1983, pp. 64-65. Tanto Körner como Butts son mencionados por Susan Feldman. Feldman sostiene que los conceptos son reglas de significado (meaning-rules) que nos permiten realizar juicios analíticos válidos, mientras que los esquemas son reglas de referencia (reference rules) que posibilitan juicios sintéticos válidos. $C f$. S. Feldman, «Kant's Schemata as Reference Rules», en G. Funke y Th. Seebohm, (eds.), Proceedings of the Sixth International Kant Congress. Washington: Center for Advanced Research in Phenomenology \& University Press of America, 1989, II, 1, pp. 229-240. Los trabajos de Körner y Feldman no son analizados en este artículo dado que no conceden importancia al concepto de interpretación.

35 Cf. A 56/B 80.

36 Cf. Z. Loparic, op. cit., pp. XXIII-XXIV. Cabe destacar que una interpretación del esquematismo trascendental a partir de la consideración de la «Analítica trascendental» como 
Loparic considera que no se debe rechazar su interpretación semántica con el argumento de que Kant presenta una teoría de la constitución a priori del mundo fenoménico. En efecto, se pueden distinguir dos tipos de semánticas. Hay una semántica realista que presupone que el dominio de interpretación es enteramente independiente de lo que es interpretado. Para esta semántica, interpretar significa asociar, de una u otra manera, conceptos o términos ya formados con objetos previamente dados. Ahora bien, no toda semántica necesita aceptar semejantes presupuestos. La semántica kantiana es una semántica constructivista, según la cual interpretar significa generar, por medio de una operación de construcción, un objeto que satisface las condiciones del concepto todavía vacío y que lo cumple. Dicho en otras palabras, la semántica constructivista, a fin de poder asociar, necesita constituir los dos términos de la asociación. ${ }^{37}$

Según Loparic, el dominio de interpretación de los conceptos representati$\operatorname{vos}^{38}$ en general es el dominio de los datos intuitivos $\left(D_{i}\right)$. Tal dominio se divide en tres subdominios de interpretación correspondientes, en cada caso, a cada uno de los tres tipos de conceptos representativos. A las categorías les corresponde el subdominio de interpretación de los esquemas puros $\left(D_{e}\right)$; a los conceptos matemáticos, el de los constructos matemáticos $(D)$; a los conceptos empíricos, finalmente, el de los fenómenos susceptibles de darse en la experiencia posible $\left(D_{f}\right)$. Ahora bien, los esquemas trascendentales son también determinaciones de los fenómenos. Por tanto, las categorías legislan asimismo sobre el dominio de la experiencia posible $\left(D_{f}\right)$. Además, las categorías de la cantidad y la cualidad legislan sobre el dominio de los constructos matemáticos $\left(D_{c}\right) \cdot{ }^{39}$

En conclusión, el esquematismo trascendental no es sino una interpretación de las categorías. Su contribución radica en la generación de modelos intuitivos correspondientes a los conceptos puros del entendimiento. Tan sólo de esta manera las categorías adquieren significado objetivo. ${ }^{40}$

Señalemos que Loparic menciona el empleo, por parte de Kant, del término «ontología» para caracterizar la filosofía trascendental. Sin embargo, considera que, según Kant, la ontología general debe ser concebida como una semántica a priori del discurso objetivamente válido acerca de la naturaleza. ${ }^{41}$

semántica trascendental también ha sido desarrollada por Daniel O. Pérez. $C f$. D. O. Pérez, Kant e o problema da significação. Curitiba: Champagnat, 2008, pp. 152-184.

37 Cf. Z. Loparic, op. cit., pp. XXV-XXVI.

38 Por «concepto representativo» entendemos los conceptos a los cuales puede corresponderles un objeto en la intuición. Esos conceptos son las categorías y los conceptos empíricos y matemáticos. Representaciones tales como las ideas de la razón, evidentemente, no forman parte de esta clase de conceptos.

39 Cf. Ibid., pp. 173-174, 188 ss.

40 Cf. Ibid., pp. 151-152.

$41 C f$. Ibid., pp. 11 ss. 
Consideramos que las interpretaciones de Butts y Loparic ponen al descubierto una de las dimensiones de la doctrina del esquematismo trascendental. En efecto, es innegable que esa doctrina procura vincular las categorías con elementos intuitivos. Por tal motivo es legítimo considerar el esquematismo trascendental desde el punto de vista de la interpretación de las categorías. Las lecturas de Butts y Loparic se ajustan en esa medida al texto kantiano. Sin dudas, la caracterización de Loparic es más acertada, en virtud de que entiende que la semántica kantiana es constructivista.

Sin embargo, a diferencia de las interpretaciones mencionadas, nosotros consideramos que la doctrina del esquematismo trascendental es el momento decisivo de la ontología kantiana. Estimamos que la preocupación fundamental de Kant en el «Capítulo del esquematismo» es poner al descubierto las propiedades ontológicas fundamentales de los objetos empíricos. Ciertamente, la producción de tales propiedades presupone que las categorías sean referidas a elementos intuitivos. Ahora bien, esa producción presupone asimismo que la multiplicidad empírica sea unificada por tales conceptos. Por tanto, abordar el esquematismo trascendental tan sólo a partir de la necesidad de que las categorías se refieran a modelos intuitivos o predicados observacionales, y no poner también el acento en la necesidad de que la multiplicidad empírica sea unificada, es tener en cuenta tan sólo uno de los aspectos de esa doctrina. No se debe considerar tan sólo la necesidad de que las categorías sean aplicadas, sino también la necesidad perentoria de que la multiplicidad empírica sea subsumida. En otras palabras, no se debe omitir el hecho de que el esquematismo trascendental está encaminado a conceder a la multiplicidad empírica una estructuración temporal análoga al contenido intelectual de las categorías.

En conclusión, pensamos que el propósito esencial del «Capítulo del esquematismo» es producir las propiedades ontológicas fundamentales de los objetos empíricos a partir de la puesta en relación de las categorías con la multiplicidad empírica, y no tan sólo conceder referencia y significado objetivo a las categorías. Por consiguiente, nuestra objeción principal contra las interpretaciones semánticas del esquematismo trascendental es que éstas son unilaterales. ${ }^{42}$ MARTíN

42 La lectura psicológica del esquematismo trascendental, si bien tiene menos asidero que la interpretación semántica, también está presente en la bibliografía crítica. Ernst Cassirer ha afirmado que la doctrina del esquematismo se ocupa del problema de la posibilidad psicológica del concepto general. Tal consideración psicológica serviría de ilustración y complemento a las investigaciones de orden lógico. $C f$. E. Cassirer, El problema del conocimiento en la filosofía y en la ciencia modernas, tr. W. Roses. México: FCE, 1956, t. II, p. 665. Más recientemente, Alexis Philonenko ha sostenido que la doctrina del esquematismo puede ser interpretada como una refutación del empirismo que se desarrolla en el mismo terreno psicológico que aquél. $C f$. A. Philonenko «Lectura del esquematismo trascendental», Ágora, 7 (1988), pp. 9-25, aquí pp. 14-17. Nuestro artículo pone en evidencia que la lectura psicológica del esquematismo tras- 
ARIAS-ALBISU es Becario Postdoctoral de CONICET (Consejo Nacional de Investigaciones Científicas y Técnicas, Argentina) en el Proyecto de Investigación Postdoctoral: Los esquemas de las ideas de la razón en Immanuel Kant. Un análisis de su naturaleza y su carácter regulativo

\section{Publicaciones recientes:}

«Los esquemas trascendentales como procedimientos y productos», en Revista de filosofía (Universidad Complutense de Madrid), en prensa.

«Una relación de homogeneidad entre términos heterogéneos. El concepto de homogeneidad en el capítulo del esquematismo de la Crítica de la razón pura», en Diánoia (Universidad Nacional Autónoma de México), vol. LIV, No 63, México D. F. (México), 2009, pp. 71-88.

Dirección electrónica: arias.martin@gmail.com

cendental es incorrecta. En efecto, los esquemas-productos son las propiedades que todos los objetos empíricos tienen que presentar necesariamente a fin de ser objetos de una experiencia humana única. Tales propiedades, por tanto, no son meros hechos mentales que descubriríamos por introspección cada vez que pensáramos en objetos. 
